\title{
O'ZBEK VA TURK TILSHUNOSLIGIDA EGA TALQINI
}

Zilola XUDAYBERGENOVA*

\begin{abstract}
Annotatsiya
O‘zbek va turk tillarining qiyosiy tadqiq etilishiga doir bir qator tadqiqotlar mavjud. Biroq bu ishlar asosan qiyoslanayotgan tillarning leksik va morfologik xususiyatlarining tadqiqini $\mathrm{O}^{6} \mathrm{z}$ ichiga oladi. O'zbek va turk tillarining sintaktik xususiyatlari, xususan, ega, eganing $0^{\prime} z b e k$ va turk tilshunosligidagi talqini va tadqiqi, ifoda materiallari solishtirilib o'rganilmagan. Zero, turk tilshunosligida va o'zbek tilshunosligida eganing talqinida tasniflanishida farqli yondashuvlar ko'zga tashlandi. O'zbek tilshunosligining rus tilshunosligi ta'sirida, turk tilshunosligining g'arb tilshunosligi, asosan fransuz va nemis tilshunosligi ta'sirida shakllanganligi natijasida gapning bosh bo'laklarini, xususan, eganing talqini va tadqiqida farqli yondashuvlarning tatbiq etilgani ko'zga tashlanadi. Turk tilshunosligida ega vazifasida kelgan so'zlar va so'z birikmalarining leksik-semantik mazmuni, sintaktik qurshovi, sintaktik vazifasi ham diqqatga olinadi. O'zbek tilshunosligida ega vazifasida kelgan so'zlar va so' $\mathrm{z}$ birikmalarining ko'pincha jumladan ajratilgan holda o'rganilganligi kuzatiladi. Bundan tashqari , o'zbek tilida eganing tuzilishiga ko'ra turlari ham tasniflangan. Bu maqolda o'zbek va turk tilshunosligidagi eganing talqin qilinishi, eganing ifodalanish usullari qiyoslab o'rganiladi, ular o'rtasidagi umumiylik va tafovutlar aniqlanadi. O'zbek tilshunosligida sintaktik birliklar tadqiqida qo'llanilgan usullarga asoslangan holda qiyoslanayotgan tillardagi ega, uning struktur turlari, ifodalanish usullari o'rganiladi. Eganing ifoda materiallari solishtirish jarayonida, bu vazifada kelgan so'z turkumlarining grammatik xususiyatlari ham tavsiflanadi.
\end{abstract}

Kalit So'Zlar: O`zbek Tili, Turk Tili, Sintaksis, Ega, Eganing Tasnifi

\section{ÖZBEK TÜRKÇESİ VE TÜRKIYYE TÜRKÇESİ DİLBILIIMINDE ÖZNENIN YORUMLANMASI}

\section{Özet}

Özbek Türkçesi ve Türkiye Türkçesinin karşılaştırmalı incelenmesine ait birkaç araştırma mevcuttur. Ancak bu çalışmalar genel olarak karşılaştırılan dillerin leksik ve morfolojik özelliklerinin incelenmesni barındırır. Özbek Türkçesi ve Türkiye Türkçesinin sözdizimsel özellikleri, bilhassa, öznenin Özbek Türkçesi ve Türkiye Türkçesinin dilbiliminde yorumlanması ve incelenmesi, ifade malzemeleri karşılaştırılarak araştırılmamıştır. Zaten Özbek Türkçesi ve Türkiye Türkçesinin dşlbşlşmşnde öznenin yorumlanmasında ve sınıflandırılmasında farklı yaklaşımlar gözlemlenir. Özbek Türkçesi dilbiliminin etkisinde, Türkiye Türkçesi dilbiliminin Batı dilbilimi, genelde Fransız ve Alman dilbilimine etkisinde gelişmiş olduğu sonucunda, özne dâhil cümlenin tüm öğelerinin yorumlanması ve incelenmasine farklı yönlerden yaklaşıldığı göze çarpar. Türkiye Türkçesi dilbiliminde özne görevinde kullanlan kelimeler ve kelime öbeklerinin anlam özellikleri, cümlenin sözdizimsel bağlamı, yüklemin özellikleri de dikkata alınır. Özbek Türkçesi sözdiziminde özne görevinde kulanılan kelimeler ve kelmeler öbekleri çoğunlukla cümleden ayırtedilmiş şekilde incelenir. Aynı zamanda Özbek Türkçesi dilbiliminde özeninin yapısı bakımından sınıflandırılması da yapılmıştır. Bu makalede Özbek Türkçesi ve Türkiye Türkçesi dilbiliminde öznenin yorumlanması, öznenin görevinde kullanılan birimler karşılaştırılarak incelenir, onların

* Prof. Dr., Bartın Üniversitesi Edebiyat Fakültesi Çağdaş Türk Lehçeleri ve Edebiyatları Bölümü Öğretim Üyesi, elmek: aloliz74@mail.ru, zkhudaybergenova@bartin.edu.tr.

ORCID ID: https://orcid.org/0000-0003-1021-3053 
arasındaki ortak ve farklı özellikler belirtilir. Özbek Türkçesi dilbiliminde kullanılan yöntemlerden yola çıkarak karşılaştırılmakta olunan dillerdeki özne, öznenin yapı bakımından çeşitleri, özne yerinde kullanılan birimlerin özellikleri araştırılır. Öznenin ifade malzemelerini karşılaştırma sürecinde bu görevi üstlenen kelime türlerinin gramer özellikleri betimlenir.

Anahtar Kelimeler: Özbek Türkçesi, Türkiye Türkçesi, Sözdizimi, Özne, Öznenin Sinıflandırilması.

\title{
INTERPRETATION OF THE SUBJECT IN UZBEK AND TURKISH LINGUISTICS
}

\begin{abstract}
There are a number of studies on the comparative study of Uzbek and Turkish languages. However, these studies include learning of the lexical and morphological features of the contrasting languages. The syntactic features of the Uzbek and Turkish languages, in particular, their interpretation and research in the Uzbek and Turkish linguistics, and the methods of expressions have not been studied. Thus, different approaches to the classification of subject in the Turkish Uzbek linguistics have been observed. Uzbek linguistics was formed under the influence of the Russian linguistics, Turkish linguistics was influenced mainly by French and German linguistics. As a result, it has been used different approaches in studying and interpretation in Uzbek and Turkish languages. The lexical-semantic content of the words and phrases of the Turkish linguistics, which were used as a subject and there syntactic position are also taken into consideration. It is observed that the words-subjects and phrasessubjects in Uzbek sentences have been studied separately from sentences. In addition, the structural types of the subject in the Uzbek language were also classified. In this article, the interpretation of the subject in Uzbek and Turkish linguistics, the ways of expression of the subject are compared, and the differences and community between them are determined. Uzbek scientific methods of the study of syntactic units are exploited in comparing subject in Uzbek and Turkish languages, its structural types, methods of expression. During comparison of the expression material of subject is characterized by their grammatical properties.
\end{abstract}

Key Words: Uzbek Language, Turkish Language, Syntax, Subject, Classification Of The Subject.

\section{Kirish}

XIX asrning ikkinchi yarmidan boshlab XX asr boshlariga qadar g'arb qiyosiy-tarixiy tilshunosligining ta'sirida mashhur ajnabiy va rus turkshunos tilshunoslari turkiy tillarni qiyosiy o'rganish sohasida ham samarali izlanishlar olib borganlar(Abdurasulov, 2008 :3). Ayniqsa, bu sohadagi izlanishlar XX asr tilshunosligida keng quloch yoydi: Rossiya fanlar akademiyasining Tilshunoslik instituti turkiy tillar bo'limi, uning Peterburg va Sibir bo'limlari tilshunoslari tomonidan turkiy xalqlar yashovchi hududlardagi barcha turkiy elat va xalq tillari yetarli o'rganildi; barcha hozirgi turkiy tillarning tovushlar tizimi, lug'at boyligi va grammatik qurilishini o'rganish bo'yicha chuqur ilmiy-tekshirish ishlari olib borildi; shu asosda hozirga qadar ko'plab ilmiy maqolalar, monografiyalar, turkiy tillarning qiyosiy lug'atlari yaratildi. Turkiy tillarning mukammal qiyosiy grammatikalari asosan XX asrning II yarmida Rossiya fanlari akademiyasi Moskva va Sankt-Peterburg tilshunoslik institutlari olimlari tomonidan yaratildi. N.A.Baskakov, B.A.Serebrennikov, N.Z.Gadjiyeva, A.M.Shcherbak kabi rus tilshunos olimlarining ko'p yillik samarali izlanishlari natijasida turkiy tillarning bir necha jildlik ilmiy tarixiy grammatikalari va monografiyalari yaratildi(Baskakov N, 1960: Serebrennikov, Gadjiyeva, 1986, Sravnitelno-istoricheskaya grammatika tyurskix yazıkov, 1984, Sravnitelno istoricheskaya grammatika tyurskix yazıkov, 1988, Shcherbak, 1970, Shcherbak, 1977, 
Shcherbak, 1981). Bu ishlar turkiy tillarning tarixiy-qiyosiy grammatikasi masalalari bo'yicha yozilgan. Hozirgi turkiy tillar, shu bilan birga, o'zbek va turk tillari qiyosi grammatikasi bo'yicha tadqiqotlar XX asrning oxirlarida, O'zbekiston mustaqillikka erishib, Turkiya bilan diplomatik aloqalar o'rnatilgandan keyin boshlangan. Bir til oilasiga mansub bo'lgan o'zbek va turk tillarining leksik, grammatik xususiyatlarini tadqiq etishga qaratilgan qator ilmiy izlanishlar, tadqiqotlar amalga oshirilgan bo'lsa-da, bu ishlarda asosan har ikki tilning leksiksemantik, morfologik xususiyatlari o'rganilgan (Usmonova, 1998; Xudoyberganova, 1999; Siddiqov, 2000; Shabanov, 2004; Abdurahmonova, 2004). Ya'ni bu sohada hali o'z yechimini topmagan masasalar oz emas. $\mathrm{O}^{\prime} z$ zbek va turk tillaridagi ega, uning talqinlari va ifoda materiallari tadqiqi masalalari shular jumladandir.

\section{O'zbek va turk tilshunosligida eganing o'rganilishi}

O'zbek tilida bosh bo'laklarning spetsifik xususiyatlari mukammal tadqiq etilgan, bu masalaga bag'ishlangan maxsus tadqiqotlar ham mavjud. Ushbu tadqiqotlarda eganing tarkibi, tuzilishi xususidagi fikr-mulohazalar bayon qilinadi (Bu haqda qarang: Shoazizov, 1973:116136). A.R.Sayfullayev gap bo 'laklarining semantik-leksik xususiyatlarini tadqiq etar ekan, egani strukturasiga ko'ra uch turga bo'ladi: sodda, qo'shma, murakkab egalar. Shuningdek, ega leksik-semantik belgilariga binoan olti guruhga ajratiladi: shaxs; predmet; belgi; miqdor; harakat-holat; o'rin-joy tushunchasini bildiruvchi egalar (Sayfullayev, 1984b: 37-42). A.Safayev o'zbek tilidagi gapning bosh bo'laklarini tadqiq etgan bo'lsa-da, tilshunos eganing tuzilishiga ko'ra turlarini farqlamagan. Chunki tilshunos gapning bosh bo'laklarini ularning ishtirok etish-emasligi nuqtai nazaridan tadqiq etgan (Safayev, 1958). Ma'lumki, o'zbek tilshunosligi rus tilshunosligi ta'sirida shakllangan bo'lib, eganing talqinida ham rus tilshunoslarining qarashlari o' $\mathrm{z}$ ifodasini topganı kuzatiladi.

Turk tilshunosligida ega kesimdan keyingi ikkinchi bo'lak bo'lib, kesimdan anglashilgan harakat-holatning bajaruvchisini bildiruvchi bo'lakdir. Ya'ni ega kesimdan anglashilgan ish-harakatning amalga oshiruvchisi, reallashtiruvchisi hisoblanadi (Dizdaroğlu, 1976:15; Demir, Y1lmaz, 2003:209-210). Xususan, egasiz gap bo'lmaydi, shaklidagi qarash natijasida grammatik ega (sözde özne), mantiqiy ega mavjudligi e'tirof etiladi (Demir, Yılmaz, 2003:209-210).

Ega tushunchasi uchun M.Ergin «fail»(Ergin, 1993:337), T.Banguo'g'lu «kimse» (Banguoğlu, 1990:526) atamalarini qo'llanganlari holda, boshqa tilshunoslar tomonidan asosan «özne» atamasining qo'llanilganligi kuzatiladi. M.Ergin ega xususida qisqacha ma'lumot keltiradi hamda ba'zi gaplarda ega bo'Imasligini qayd etadi(Ergin, 1993:337). T.Banguo'g'lu nisbatan ilgari ketadi va eganing grammatik ega, mantiqiy ega kabi turlarini ko'rsatadi(Banguoğlu, 1990:526). Biroq turk tilshunoslarining barchasi ham, masalan, L.Karaxan, E.Yaman kabilar egani guruhlashtirmaydi(Karahan, 2006:43; Yaman, 2000:225). Ayni paytda E.Yaman ba'zi gaplarning egasi yashirin bo'lishini bildiradi (Yaman, 2000:225). V.Xatibo'g'lu turk tilidagi eganing quyidagi o'n bir turini ko'rsatadi: haqiqiy ega (gerçek özne), nutqiy ega (sözde özne), yashirin ega (örtülü özne), noaniq ega (belirsiz özne), umumiy ega (ortak özne), kuchaytirilgan ega(pekiştirilmiş özne), bog'lovchili ega (bağlaçlı özne), izohlovchili ega (açıklayıcıdan özne), undalmali ega (seslenmeli özne), moslashuvchi ega (kayan özne), -dır affiksli ega (bildirme koşaçlı özne) (Hatiboğlu, 1982:110-120). H.Dizdaro'g'luning ishida eganing to'qqiz shakli tasniflanganligi kuzatiladi. Bunda V.Xatibo'g'lu tomonidan farqlangan noaniq ega, moslashuvchi ega, -dır -tür affiksli ega, bog'lovchili ega ayni tasnifdan o'rin olmagan, takroriy ega (yinelenmiş özne), qoliplangan ega (kalıplaşmış özne) tiplari esa kiritilgan(Dizdaroğlu, 1976).

Albatta, eganing takroriy qo'llanishi, uyushgan kesim uchun yagona, umumiy eganing bo'lishi, grammatik, mantiqiy ega singari tushunchalar o'zbek tilshunosligida ham mavjud, biroq faqat eganing tuzilishi, gapda eganing grammatik shakllangan-shakllanmaganligiga ko'ra ega turlarini tafovutlash, nazdimizda, yetarli emasday ko'rinadi. Masalan, undalmaning gapda 
sintaktik vazifani bajarmasligi, biron-bir gap bo 'lagi bo'la olmasligi ayon. Biroq turk tilida undalmani yoki undov so'zli undalmani eganing bir shakl turi - undalmali ega deb ta'riflanganini ko'rish mumkin. Shu fikrga ko'ra quyidagi gapning egasi ey tatlı gece -ey totli tun birikmasidir:

\section{Ey tatl gece yillarca devam et - Ey totli tun, yillar bo 'yi davom et.}

Bu gapning egasi sen olmoshi bo'lib, u yashiringan, ya'ni egani kesimga savol berish orqali topish mumkin. Demak, keltirilgan gap aslida bir bosh bo'lakli gapdir. Ega tarzida ko'rsatilgan birikma gapning undalmasidir. Demak, V.Xatibo'g'lu va H.Dizdaro'g'lu taklif etgan ega tasnifi til hodisalarini nisbatan boshqacha me'yorlar asosida tadqiq etish, ayrim sintaktik hodisalarni o'zgacha baholash natijasi sifatida yuzaga kelgan. Xususan, ularning tadqiqotlarida ayrim leksik-semantik, morfologik, sintaktik, stilistik me'yorlarning aralashib ketgan ko'rinadi.

O'sman Bo'lulu egani mavjud bo'lgan tasniflardan birmuncha boshqacha tartibda guruhlarga ajratadi. Bunda eganing o'n turi qayd etiladi: 1) bajaruvchi ega(yapıcı özne) tub fe'l bilan ifodalangan kesimning bajaruvchisini bildiradi; 2) ta'sirlovchi ega (etkileyici özne)ning kesimi o'timli fe'llar bilan ifodalanadi; 3) ta'sir o'tkazmaydigan ega (etkisiz özne) kesimi o'timsiz tub fe'llar bilan ifodalangan gaplarning egasidir; 4) egasiz gaplar(öznesizlik egasizlik)ning kesimi -(y)ıl, -(1)n affikslari bilan yasalgan fe'llar bilan ifodalanadi. $\mathrm{Bu}$ o'rinda majhul nisbatdagi fe'l bilan ifodalangan kesimli gaplar e'tiborda tutiladi; 5) ta'sirlangan ega(etkilenen özne) kesimi o'zlik nisbat shaklidagi gapning egasi; 6) birgalik egali(imeceli özne) gap kesimi vazifasida birgalik nisbatdagi fe'llar qo'llaniladi; 7) bajartiruvchi ega(yaptırıcı özne) o'timlidan o'timsizga aylangan fe'l bilan ifodalangan kesimning egasidir. Masalan, "Anne çocuğu bahçede gezdirdi - Ona bolasini bog 'da kezdirdi» gapida harakat bajaruvchi ona emas, boladir; 8) bavosita ega(aracılı özne); 9) tazyiq o'tkazuvchi ega(baskıcı özne); 10) majburlovchi ega(zorlayıc1 özne) (Bolulu, 1992:24-29, 46-52). O‘.Bo'lulu tarafidan tilga olingan ega turlaridan so'nggi uchtasi orttirma nisbat shaklidagi fe'llarga taalluqli. Tazyiq o'tkazuvchi hamda majburlovchi ega turlari birdan ortiq orttirma nisbat ko'rsatkichini olgan fe'lning kesim vazifasida ishlatilishi natijasida farqlangan. O'.N.Tunaning fikricha, darll - xafa bo'l, eksil kamay kabi fe'llar ta'sirlangan egali gapni hosil qiladi (Tuna, 1986:420). Bu holatning sababini quyidagicha izohlash mumkin: turk tilshunosligida fe'lning nisbat shakllari grammatik kategoriya mavqeiga ega emas. Orttirma, birgalik, majhul, o'zlik nisbati ko'rsatkichlari fe'l yasovchi qo'shimchalar sifatida qabul qilinadi. Natijada bu nisbatlar ko'rinishidagi fe'llar yasama fe'llar hisoblanadi. Nisbat shakllari orqali yuklanadigan ma'nolar esa valentliklar yoki eganing turlari sifatida talqin etiladi. Shunday bo'lsa-da, ayrim hollarda -ır, -ar, -gır kabi orttirma nisbat qo'shimchalari o'zining so'z yasash faolligini yo'qotganligi qayd etiladi (Erdal, 1991).

O'Demirjan turk tilida fe'lning tuslanish tizimini tadqiq etar ekan, fe'lning nisbat shakllariga teng keluvchi grammatik ko'rsatkichlar vositasida yuklanuvchi valentliklar masalasini tahlil qiladi. O'.Demirjan U.Bo'lulu singari fe'l nisbatlarining ko'rsatkichlari vositasida gapdan anglashilgan voqea-hodisa ishtirokchilarini ifodalaydigan gap bo'laklarini tadqiq etadi. Xususan, fe'lning nisbatlari asosida bajaruvchi, bajartiruvchi, yuzaki bajartiruvchi, vositachi-bajartiruvchi, majburlovchi ega turlarini farqlaydi (Demircan, 2003:11-17). R.Anderxill ham nisbat ko'rsatkichlari yangi ega turini yaratadi, deya e'tirof etadi (Underhill, 1976).

\footnotetext{
Qiyoslang:

Herkes oturdu (Hamma o'tirdi)> Hasan herkesi masaya oturttu (Hasan hammani stolga o'tqazdi).
}

Ushbu gaplarda nisbat ko'rsatkichlarining qo'llanilishi natijasida harakat bajaruvchi ega o'zgargan. 
M.Bilgin turk tilidagi eganing to'rt qo'llanish shaklini ajratadi: umumiy ega, kuchaytirilgan ega, izohli ega, murojaatli ega (Bilgin, 2006:459-461). Umumiy ega, boshqa tilshunoslar qayd etganidek, uyushgan kesimlarning umumiy egasidir. O‘zlik olmoshi yoki ki yuklamasi ishtirokidagi, xususan, takroriy shaklda qo'llangan ega kuchaytirilgan ega turini yuzaga keltiradi. Izohli ega o'zbek tilidagi ajratilgan egaga, murojaatli ega o'zbek tilidagi undalmaga teng keluvchi sintaktik birlikdir.

Demak, turk tilidagi eganing tasniflanishida ikki yo'nalish ko'zga tashlanadi: birinchidan, ayrim sintaktik hodisalarni farqlamaslik, stilistik hodisalarni sintaksis doirasiga kiritish asosida amalga oshirilgan ega tasniflari (V.Xatibo'g'lu, H.Dizdaro'g'lu, M.Bilgin); ikkinchidan, fe'lning nisbat shakllari asosida taqdim etilgan ega tasniflari (U.Bo'lulu, $\mathrm{O}^{`}$.Demirjan). Eganing ikkinchi tipdagi tasniflari transformatsion grammatikaning ta'siri natijasida yuzaga kelgan. Umuman olganda, turk tilshunosligida $\mathrm{g}^{\mathbf{}}$ arb tilshunosligining ta'siri, ayniqsa, fransuz ve nemis tilshunoslarining qarashlari izlari kuzatiladi.

Yuqorida bayon etilgan fikr-mulohazalar asosida quyidagi xulosaga kelish mumkin: O'zbek va turk tillarida sodda gapning bosh bo'laklaridan biri bo'lgan ega har ikki tilda deyarli bir xil ta'riflansa-da, ammo ular turlarining ajratilishi(turk tilida), ifoda materiallarining sharhlanishiga ko'ra tafovutlanadi. Turk tilshunoslari tomonidan taqdim etilgan ega tasniflarida morfologik tasnif va tahlillardagi farqli qarashlarning sintaksisga ham bevosita ta'sir ko'rsatganligi, ba'zi stilistik hodisalar sintaktik hodisa sifatida baholanganligi, tamomila boshqa sintaktik hodisa ega turi deb ajratilganligi, xususan, ayrim adabiyotlarda transformatsion grammatika me'yorlari asosida yangi ega turlari tasniflanganligi ayon bo'ladi. Birinchi holatda, avval ta'kidlanganidek, ega turlari ba'zi sintaktik, stilistik hodisalarning aralashtirib yuborilishi natijasida tasniflangan bo'lsa, ikkinchi holatda fe'l bilan ifodalangan kesimning nisbat shakllari eganing yangi turlari farqlanishiga sabab bo'lgan. Zero, mantiqiy sub'ekt har ikki tilda umumiy bo'lishi mumkin, ammo ularni ifodalovchi vositalar o'rtasida tafovutlar kuzatiladi. Shu bois, o'zbek va turk tillaridagi eganing universal va relevant belgilarini aniqlash uchun gap bo'laklarining tuzilishiga ko'ra tasnifiga asoslanish qulay.

\section{Eganing tuzilishiga ko'ra turlari va ularning ifoda materiallari}

Solishtirilayotgan tillardagi eganing bevosita qiyosiy tadqiqini olib borish uchun bu gap bo'laklarining tuzilishiga ko'ra uch tipi asosida ish ko'rish maqsadga muvofiq: sodda ega, qo'shma, murakkab ega. Sodda ega faqat bosh kelishik formasida shakllanib, ega funksiyasida qo'llaniladigan, belgisi kesim tomonidan aniqlanadigan predmet (substantiv) yoki predmetlashgan - predmet tasavvuridagi (substantivlashgan) bo'lakdir (Omonturdiyev, 1988: 74). Taqqoslanayotgan tillardagi sodda egalar quyidagicha ifodalanadi:

* ot bilan. Bunda otning barcha ma'no turlari - atoqli, turdosh, mavhum, aniq va sh.k. otlar gapning egasi vazifasini bajarib keladi. Masalan, quyidagi gaplarning harakat bajaruvchilari mavhum ot (hayot) va atoqli ot (Cavidan) bilan ifodalangan:

Hayot meni doim boshi berk ko 'chaga tiqadi(O.M.).

Yaşam beni hep çıkmaz sokağa sokar.

Cavidan misafirimi civarda gezmeğe götürüyor(R.N.G.).

Javidan mehmonimni sayr qilishga olib ketyapti.

* olmosh bilan. Har ikki tildagi ot xarakteridagi yoki otlashgan olmoshlar gapning egasi bo'lib keladi. Masalan, keyingi gaplarda harakat bajaruvchi kishilik olmoshi (men) va otlashgan ko'rsatish olmoshi (bunlar - bular) vositasida anglashilgan:

Men baxtni sandig i bilan sizga keltirib berar edim(O.Yo.).

Ben mutluluğu sandiğ ile size getirecektim. 
Fakat bunlar benim ihtiyar kafamın alacă̆ı şeyler değil (R.N.G.).

Bular menday keksa odamning aqli yetadigan narsalar emas.

* harakat nomi bilan: O‘zbek tilida -(i)sh, -moq, -maslik shaklli harakat nomlari bilan ifodalangan egalar faol qo'llaniladi.

Masalan:

Bu gapga ishonish qiyin!(O.M.)

Bu lafa inanmak zor.

Bir qizni bekor malomatdan qutqarmoq ham yigitning ishi(O.M.).

Bir kızı boş aşağılamadan kurtarmak da yiğidin işi.

Endi bunday odamning topilmasligi - boshqa masala(O.M.).

Şimdi böyle bir insanın bulunmaması - başka konu.

Turk tilida esa harakat nomi -mak, -mek; -ma, -me; -(y)ış, -(y)iş, -(y)uş, -(y)üş affikslari bilan yasaladi(Korkmaz, 2003:864). Bu qo'shimchalardan birinchi ikkitasi yordamida hosil qilingan harakat nomining ega vazifasida ishlatilishi uchinchisiga nisbatan ancha faol:

Yaşamak, yaşamak bir alçaklıktır(Y.K.K.).

Yashamoq, yashamoq bir pastkashlikdir.

Genç klzın açıkça halini söylemesi Ali Riza Bey'e dokundu(R.N.G.).

Yosh qizning holini ochiq aytishi Ali Rizobeyga qattiq ta 'sir qildi.

Önce ağlayan çocukların sesine yeni bir ăglayış katıldı(R.N.G.).

Avval yig lashni boshlagan bolalarning ovoziga yana bir yangi yig'i(yig'lash) ovozi qo'shildi.

-ma harakat nomi yasovchisi umuman qadimgi turkiy tilga oid manbalarda -ma(-ba) ko'rinishida uchrasa-da, hozirgi o'zbek tilida ot yasovchi sifatida qo'llaniladi (birlashma, suzma) (Serebrennikov, Gadjiyeva,1979: 226-227). -ma, -me affiksining -mak, -mek qo"shimchasi tarkibidagi $\mathbf{k}$ undoshining unlilar o"rtasida kelib, ular ta'sirida "edirilib" yo"q bo"lib ketganligi orqali hosil bo'lganligi xususida ham fikrlar ham mavjud (Korkmaz, 2003:882).

-mak, -mek; -ma, -me affiksli so'zlar ega vazifasini bajarishga xoslanganida, ular o'rtasida quyidagi farq kuzatiladi. Ega vazifasidagi -mak, -mek shaklli harakat nomlarida ishharakat, holat ma'nosi saqlanadi, ega vazifasidagi -ma, -me qo'shimchali leksemalarda esa harakat nomlanadi, ya'ni bu qo'shimcha vositasida mavhum otlarga yaqin semali so'zlar hosil qilinadi. Masalan, quyidagi gapning egasi bo'lib kelgan harakat nomi ko'proq otga teng keluvchi semaga ega, shuning uchun tarjimada ekvivalent shakldagi harakat nomi emas, ot qo'llangan:

Omuzlarında ve kollarında başlayan bir titreme bütün vücudunu sarsmaya başlamıştı(Y.K.K.).

Yelkalaridan va qo 'llaridan boshlangan titroq butun vujudini larzaga solmoqda edi.

Turk tilidagi -(y)ış, -(y)iş, -(y)uş, -(y)üş affikslari semantik jihatdan -ma, -me qo'shimchalariga o'xshashdir. Bu ko'rsatkichlar fe'lning bosh ma'nosini - harakat-holat semasini o'zgartirmay, uni otlashtiruvchi, unga nom beruvchi ko'rsatkichlardir. Agar -ma, me'li fe'l ko'pincha harakatni nomlasa, -(y)ış, -(y)iş, -(y)uş, -(y)üsş'li leksema ish-harakatni amalga oshirish tarzini ifodalash imkoniga ega. Shu sababli, -(y)ış, -(y)iş, -(y)uş, -(y)üş ko'rsatkichli harakat nomi boshqa tipdagi harakat nomlariga qaraganda ega vazifasida kam 
ishlatiladi. Shuningdek, o'zbek tilidan farqli ravishda turk tilidagi harakat nomlarining barcha ko'rsatkichlari bo'lishsizlik elementini olib ega vazifasida keladi. Bunda harakat nomi affikslari quyidagi ko'rinishga ega bo'ladi: -mayış, -meyiş; -mamak, -memek; -mama, -meme.

Masalan:

\section{Beğenmemek mümkün mü?(R.N.G.)}

\section{Yoqtirmaslik mumkinmi?}

Demak, solishtirilayotgan tillardagi ega vazifasidagi harakat nomlari yasovchilari fonetik jihatdan muqobil bo'lsa-da (-(i)sh -- -(y)ış, -(y)iş, -(y)uş, -(y)üş; -moq -- -mak, -mek), ushbu ko'rsatkichlar semantik-funksional jihatdan to'la muqobillikni saqlay olmaydi. Qiyoslanayotgan tillarda fonetik muqobillari mavjud bo'lmagan, o'zbek tilidagi -(u)v va turk tilidagi -ma, -me harakat nomi yasovchilari semantik xususiyatlariga ko'ra ekvivalent bo'lishiga qaramay, birinchi ko'rsatkichli leksemaning ega vazifasida qo'llanilishi deyarli uchramaydi. Harakat nomi yasovchilaridan -(i)sh va -mak, -mek affikslari bilan yasalgan so 'zlar ega vazifasini bajarganida, semantik, funksional jihatdan to'la muqobil keladi.

* otlashgan sifat bilan: Har ikki tildagi asliy sifatlar ham, nisbiy sifatlar ham otlashib, ega vazifasini bajarib kelishi mumkin:

Lekin o ziga juda ham erk berib yomon yo 'lga kirib ketgan tantiqlar ham bor(O.Yo.).

Ama kendini çok serbest his ederek kötü yola sapan şımarıklar da var.

Küçüklerin arasında kocaman gençler vardl(R.N.G).

Kichiklar orasida kap-katta yigitlar (yoshlar) ham bor edi.

* otlashgan sifatdosh bilan: O'zbek tilida, ma'lumki, -gan, -yotgan, -a/-y+-digan affiksli sifatdoshlar faol, -r/-mas, -vchi, -rlik, -gulik shaklli sifatdoshlar nisbatan nofaol hisoblanadi (O'zbek tili grammatikasi, 1975:510-515). Turk tilida esa -mış, -miş, -muş, -müş; dık, -dik, -duk, -dük, -tık, -tik, -tuk, -tük; -(y)an, -(y)en; -r/-maz, -mez; -(y)acak, -(y)ecek; (y)ıcı, -(y)ici, -(y)ucu, -(y)ücü affikslari sifatdosh yasovchilardir (Banguoğlu, 1990:422-427). $\mathrm{Bu}$ qo'shimchalar o'zbek tilidagi sifatdosh yasovchilar bilan paradigmatik mazmuniga ko'ra quyidagi tartibda muvofiqlashadi: -gan// -mış, -miş, -muş, -müş; -dık, -dik, -duk, -dük, -tık, tik, -tuk, -tük, -yotgan// -(y)an, -(y)en; -a/-y+-digan//-(y)acak, -(y)ecek; -r/-mas// -r/-maz, mez; -vchi/-(y)ıcı, -(y)ici, -(y)ucu, -(y)ücü. Biroq o'zaro vazifasi va ma'nosiga ko'ra ekvivalent bo'lgan mazkur shakldagi sifatdoshlar ega vazifasida kelganida, ushbu ekvivalentlikni saqlab qola olmaydi. O'zaro ekvivalent sifatdosh yasovchilari bilan yasalgan so'zlarning ega vazifasida qo'llanilishini qiyosan tahlil etaylik: o'zbek va turk tillaridagi o'tgan zamon sifatdoshlari -gan/mış, -miş, -muş, -müş; -dık, -dik, -duk, -dük, -tık, -tik, -tuk, -tük affikslari yordamida yasaladi, ya'ni bir qarashda ushbu affikslar o'zaro ekvivalentdek ko'rinadi. Ammo misollar tahliliga ko'ra quyidagi holat kuzatiladi: -dık, -dik, -duk, -dük, -tık, -tik, -tuk, -tük shaklli fe'l gapning egasi bo'lishi uchun albatta egalik qo'shimchasini olgan bo'lishi shart.

Masalan:

Asıl duyduğumuz içimizde yaşayan bir geçmiş zamanıdır (R.N.G.).

Asl uyg 'ongan hislarimiz qalbimizda yashayotgan o 'tmishimizdir.

Mazkur gapda duyduk - his etgan sifatdoshi birinchi shaxs ko'plik ko'rsatkichini olishi natijasida otlashgan va gapning egasi vazifasini ado etgan. O'zbek tilidagi -gan shaklli sifatdosh ega bo'lib kelganida, egalik ko'rsatkichini olgan bo'lishi shart emas. Masalan, keyingi gaplarning birinchisida sifatdosh egalik shaklida qo'llangan, ikkinchisida, aksincha, egalik shaklida emas:

Besh yil avval uchrashmaganimiz menga alam qiladi(O.Yo.). 
Beş sene önce görüşmediğimizden çok üzgünüm.

Bu gapni eshitganlar kulib yuborishdi(O.M.).

Bu lafi dinleyenler gülüverdiler.

Sodda fe'l-kesimlar haqida so'z ketganida, -gan va -mış, -miş, -muş, -müş affikslarining ekvivalent bo'la olishi qayd etilgan edi. Mantiqan qaralganida, ushbu qo'shimchalar sifatdosh yasovchisi sifatida ham muqobil kelishi kerak edi, biroq turk tilidagi mış, -miş, -muş, -müş shaklli sifatdoshlar ega vazifasi bajarmaydi. Gapning harakat bajaruvchisini bildiruvchi -gan shaklli sifatdoshning turk tilidagi ekvivalenti sifatida -(y)an, (y)en affiksli sifatdoshlar ham ishlatiladi. -(y)an, -(y)en qo'shimchasi aslida hozirgi zamon sifatdoshi yasovchisi bo'lib, -yotgan shaklli tuslanishsiz fe'lga mazmunan muvofiq kelsa-da, kontekstga ko'ra o'tgan zamonga oidlikni bildirishi mumkin, bu holda, tabiiyki, -gan yasovchisining ekvivalentiga aylanadi:

Dört beş yll önce bu evde yaşayanlar mutlu kişilerdi(R.N.G.).

To 'rt-besh yil avval bu yerda yashaganlar baxtli insonlar edi.

Bu gapdagi sifatdoshning o'tgan zamonga taalluqlilik ma'nosi dört beş yıl önce - to'rtbesh yil avval payt holidan anglashiladi. -(y)an, -(y)en shaklli sifatdoshlar ega vazifasini bajarganida -yotgan qo'shimchali sifatdoshga xos mazmunni reallashtiradi.

Masalan:

Telaşlı telaşlı saatine bakanlar ve ikide bir yüksekçe bir yere çıkıp uzaktan yolu gözetleyenler var(R.N.G.).

Tez-tez soatiga qarab qo'yayotganlar va ora-chora balandroq joylarga chiqib, uzoqdan yo 'Ini kuzatayotganlar bor.

Shu jihatdan, -yotgan va -(y)an, -(y)en sifatdosh yasovchilari o'zaro ekvivalent bo'ladi. Masalan: Ayni shu maqsadda yashayotganlar oz emas. Taqqoslanayotgan tillarda kelasi zamon sifatdoshlari -a/-y+-digan//-(y)acak, -(y)ecek affikslari yordamida yasaladi hamda gapda otlashib, harakat bajaruvchini ifodalaydi.

Masalan:

Aytadiganlaringiz muhimmidi?

Konuşacak olduklarınız önemliymdi?

Yarın yola çıkacaklar sabah saat sekizden önce terminale gelmiş olmalıdır(R.N.G.).

Ertaga yo 'lga chiqadiganlar soat sakkizdan avvad terminalga kelgan bo lishlari kerak.

Shu bilan birga, ega vazifasidagi -(y)acak, -(y)ecek shaklli sifatdoshlar -moqchi bo'Imoq tuzilmali sifatdoshlar bilan semantik ekvivalentlik munosabatiga kirishishi hamda harakat bajaruvchilarni maqsadi, niyati kabi ma'nolar asosida anglatishi mumkin:

Bugün sinava girecekler üst kattaki dersanede toplansin(R.N.G.).

Bugun imtihonga kirmoqchi bo'lganlar yuqori qavatdagi darsxonada to 'plansin.

Demak, o'zbek va turk tillaridagi paradigmatik jihatdan muqobil keluvchi sifatdosh yasovchilari eganing ifoda materiali ko'rsatkichi sifatida bir-biriga to'la semantik, stilistik ekvivalent bo'la olmaydi. Bir qarashda umumiylikka ega bu qo'shimchalar ma'no doirasi, qo'llanilish o'rniga ko'ra butunlay boshqa tartibdagi ekvivalentlikni namoyon qiladi. Masalan, ega vazifasida kelgan -gan shaklli sifatdoshning semantik-stilistik muqobili -dık, -dik, -duk, dük, -tık, -tik, -tuk, -tük; -(y)an, -(y)en shakliga ega, holbuki, turk tilidagi o'tgan zamon sifatdoshlari -dık, -dik, -duk, -dük, -tık, -tik, -tuk, -tük; -mış, -miş, -muş, -müş affikslari bilan yasaladi, -(y)an, -(y)en hozirgi zamon sifatdoshining yasovchisi hisoblanadi. Ayni 
chog'da, -dık, -dik, -duk, -dük, -tık, -tik, -tuk, -tük ko'rsatkichli sifatdoshning ega vazifasida kelishi uchun, o'zbek tilidagi ekvivalentidan farqli holda, uni egalik shaklida qo'llash talab etiladi. -rlik, -gulik shaklli sifatdoshlarning gapda ega vazifasini bajarishi kuzatilmadi.

* otlashgan sonlar bilan. Masalan, quyidagi gaplarda otlashgan tartib sonlar harakat bajaruvchini tartibiga ko'ra belgisini ifodalash orqali anglatgan:

Ikkinchisi hov anavi yerda yotibdi(O.M.).

Ikisi de işte orada yatiyor.

Fakat ilk sözünden sonra bir ikincisi, bir üçüncüsü lakırdıya karıştı (R.N.G.).

Ammo ilk gapidan so'ng avval ikkinchisi, keyin uchinchisi suhbatga qo 'shildi.

Turk tilshunosligida son alohida so'z turkumi sifatida farqlanmagan. Predmetning miqdorini, tartibini bildiruvchi so'zlar sifatning ma'no turlaridan biri tarzida, ya'ni son sifatlari deb o'rganiladi (Banguoğlu, 1990:351-356). Xususan, o'zbek tilida jamlovchi sonning yasovchilari bo'lmish -ov, -ovlon, -ala affikslarining muqobillari turk tilida yo'q. Bu vaziyatda sanoq sonlarga murojaat qilinadi.

Masalan:

Modern ve bazı güzel meyvelerle tertip edilmiş olan bir sofra başında dördü birlikte $\operatorname{oturdular}(\mathrm{E} . \mathrm{N}$.$) .$

Zamonaviy ko'rinishda va ayrim ajoyib mevalar bilan tuzalgan dasturxon atrofiga to'rtovi birgalikda o'tirishdi.

$\mathrm{Bu}$ esa, turk tilidagi sonlarning semantik va qo'llanilish doirasi nisbatan kengligini ko'rsatadi

* o o zbek tilida egalar otlashgan modal so 'zlar bilan ifodalanadi:

Bu bolalarning mendan boshqa hech kimga keragi yo 'q(O.M.).

Bu çocuklar ben hariç kimseye gerekmiyor.

Turk tilshunosligida modal so'zlar alohida so'z turkumi sifatida tafovutlanmagan. Umuman, modal so'zlar sirasiga kiruvchi so'zlarning biron so ' $z$ turkumi guruhiga kiritilgan hollari turk tilshunosligiga oid ilmiy manbalarda uchramadi. Lekin modal so $z$ sifatida ajratilishi zarur bo'lgan so'zlar, o'zbek tilidagichalik ko'p bo'lmasa-da, mavjud. Masalan, zarur, kerak, shart, darkor, lozim kabi so'zlar uchun turk tilida gerek, lazım so'zlari qo'llaniladi. Bunga sabab esa turk tilida fe'ldan anglashilgan ish-harakatning bajarish zarurligi, kerakligini bildiruvchi -malı, -meli ko'rsatkichli keraklilik maylining, shuningdek, gerekmek /kerak bo'Imoq fe'lining mavjudligidir. Turk tilidagi modal so'zlar ega vazifasida qo'llanmaydi.

* otlashgan ravish bilan:

Ichkari ham yomon emas(E.A.).

Içerisi de kötü değil.

Lakin mevsim icabı oralar, pekçok kimselerin rağbet ederek gittikleri ve toplandıkları yerdi(E.N.).

Lekin mavsum talabi bilan o'sha yerlar juda ko 'pchilikning rag 'bat ko 'rsatib borgan va to 'plangan joylari edi.

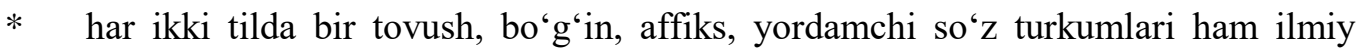
uslubdagi matnlarda ega bo'lib kelishi mumkin:

-lar ko 'plik qo 'shimchasidir. 
-ler çoğul ekidir.

O‘zbek va turk tillaridagi qo'shma ega quyidagi tuzilishga ega bo'ladi:

O'zbek va turk tillaridagi «etakchi komponent + yordamchi fe'l» tipidagi fe'llar bilan ifodalangan gap bo'laklarini o'rganish natijasida ma'lum bo'ldiki, o'zbek tilida yordamchi fe'lning barcha ma'no turlari - ko'makchi, yordamchi, to'liqsiz fe'llarning qo'shma ega tarkibida faol ishtirok etishi kuzatiladi. Turk tilida esa asosan yordamchi fe'llar qatnashuvidagi qo'shma egalar qo"llanilishi ko'rinadi. Masalan, quyidagi gapning egalari ot va yordamchi fe'llardan (qilmoq, olmak) tashkil topgan qo'shma fe'llar bilan ifodalangan:

Ha, pochchamizni xafa qilganimiz yoqmadimi?(S.A.)

Evet, eniştemizi üzdüğ̈̈müz hoşunuza gitmedimi?

Benim ona minnettar olmam lazım gelir(R.N.G.).

Men undan minnatdor bo 'lishim kerak.

Turk tilida ko'makchi fe'lli qo'shma fe'llarning ega vazifasida qo'llanilishi o'zbek tilidagidan anchagina nofaol. Masalan, quyidagi gaplarning egalari «etakchi fe'l + ko'makchi fe'l» tuzilishiga ega:

Bu bosqichlardan allaqachon o'tib ketganlar juda ko ' $p$ (O.Yo.).

Bu evrelerden çoktandır geçmiş olanlar çoktur.

A ğza geleni gelişigüzel söyleyivermek terbiyesizliktir(Ö.S.).

Og 'izga kelgan gapni pala-partish gapiraverish tarbiyasizlik alomatidir.

O'zbek tilida egalar ot, ot xarakteridagi so'zlar va ekan to'liqsiz fe'li birikuvi orqali ifodalanishi mumkin. Ega tarkibida ishtirok etadigan ekan to 'liqsiz fe'li uchun turk tilida yordamchi fe'llar yoki muayyan qo'shimchalar ishlatiladi. Masalan, quyidagi gapning egasi «baxtli ekanligi» birikmasi bilan ifodalangan bo'lib, tarjimada -lık yasovchisi yordamida yasalgan ot yoki olmak yordamchi fe'li vositasida ayni ma'no anglashilishi mumkin:

Baxtli ekanligi ko 'zidan ayon.

Mutluluğu(mutlu olduğu) gözlerinden belli.

Ikki va undan ortiq mustaqil so'zlarning birikishidan tashkil topgan, gap bo'lagi funksiyasida bir butun holda qo'llanilib, hozirgi tilda qisman leksikalizatsiyalashgan, frazeologiyalashgan leksik qo'shilmalar va tarkibi mustaqil hamda yordamchi komponentlarga ajralmaydigan sintaktik birliklar gapning kim yoki nima haqda ekanini bildirib kelsa, murakkab ega hisoblanadi. Binobarin, ikki va undan ortiq mustaqil leksik ma'noli so'zlar kompleksidan tarkib topgan, komponentlari ma'lum gap tarkibidagina o'zaro zich bog'lanib yaxlit butunlik tashkil etgan va muayyan kontekstda u yoki bu gap bo'lagi funksiyasida qo'llaniladigan sintaktik butunliklar - ajralmas sintaktik konstruksiyalar uchun murakkab bo'lak atamasini qo'llash ma'qul (Abdullayev, 1969:3).

Solishtirilayotgan tillarda murakkab ega quyidagicha ifodalanadi: Turg'un, barqaror ismlar, geografik, onomastik atamalar, tashkilot, muaassasa, kitob, jurnal nomlari gapning sub'ekti vazifasini bajaradi:

"Xalq so'zi» - boy berilmaydigan imkoniyat(G.).

“Halk Sözü̈”- elde tutulmast gereken olanak.

BM Güvenlik Konseyi önceki günkü toplantısında tazminat miktarını azaltmayı kararlaştırdı (R.H.K.). 
BM Xavfsizlik Kengashi avvalgi kuni bo 'lib o'tgan majlisida kompensatsiya miqdorini ko 'paytirishga qaror qildi.

Keltirilgan gaplarning birinchisida gazeta nomi, ikkinchisida muassasa nomi ega vazifasida qo'llangan.

$\mathrm{O}^{‘}$ zbek va turk tillaridagi frazeologik birikmalar bilan ifodalangan egalar murakkab ega hisoblanadi. Keyingi gaplarning egalari -lik qo'shimchasini olgan ko'zga yaqin, sifatdosh shaklidagi elini kolunu bağlamak - qo' ${ }^{`} \operatorname{loyog}^{`}$ ini bog'lamoq iboralari bilan ifodalangan:

Ko'zga yaqinlik ham bir balo ekan (O.Yo.).

Güzellik de bir belaymıs.

Elini kolunu bağlayan yok ya(R.N.G.).

Qo'l-oyog'ingni bog'layotganlar yo ' $q$ - $k u$.

O‘zbek va turk tillarida gapda ajralmas sintaktik konstruksiyaga aylangan aniqlovchili birikmalar murakkab ega vazifasini bajaradi:

Sherning yuragi bor senda(O.Yo.).

Aslanın kalbı var sende.

Son zamanlarda adamcă̆ızın dertlerı tazelenmeğe başladı(R.N.G.).

So 'nggi paytlarda bechora odamning dardlari yangilana boshladi.

Mazkur gaplarda «qaratqich+qaralmish» tipidagi aniqlovchili birikmalar gapning egasi vazifasini bajargan. «Ega+kesim» formasida shakllanib, gapda bitta bo'lak vazifasida keladigan qo'shilmalar(polupredikatlar) gapda murakkab ega bo 'lib keladi.

Masalan:

Sattor, oilangiz borligi menga bunchalik ta'sir qilmasligi kerak edi(O.Yo.).

Sattar, eşiniz var olduğu beni bu kadar etkilememesi lazımdı.

Aralarında bü̈yük fark olduğu meydandadır(E.N.).

Ular o'rtasida katta farq borligi ma'lum.

Teng bog'lanishli qo'shilmalar taqqoslanayotgan tillarda murakkab ega vazifasini bajarib keladi:

Marjonoy bilan Oltinoy vaqtni o 'tkazish niyatida xamir qorishdi, non yopishdi(O.Yo.).

Mercanay ve Altınay zaman geçsin diye hamur yaptılar, ekmek pişirdiler.

Vefik Paşa ile Cavidan şaşırıp kalmışlardı(R.N.G).

Vafik poshsho bilan Javidan hayron bo 'lib qolgan edilar.

Ega vazifasini bajarib kelgan ism-familiyalar tuzilishiga ko'ra murakkabdir:

Nodirning yonida ishxonalarining boshlig 'i Fayzulla Beknazarov ham shampan ichib salqinlab o'tirgan ekan(O.Yo.). dinleniyormuş.

Nadir'in yanında iş yerinin patronu Feyzulla Beknazarov da şampuan içerek

Yahya Kemal Nasif Bey Fikret' in lisanlyla serlevhal,, mevzulu şiirler de yazıyor(Ö.S.).

Yahyo Kamol Nasifbey Fikrat usulida turli mavzularda she'rlar yozyapti. 
O'zbek tilidan farqli ravishda turk tilidagi ega vazifasini bajargan so'zlar -dır, -dir, dur, -dür,-tır, -tir, -tur, -tür affiksini olgan bo'lishi mumkin. Bu holat turk tilida keng tarqalganligi sababli alohida eganing turi sifatida ajratilishiga ham sabab bo'lgan (Hatiboğlu, 1982:120). Ammo -dır, -dir, -dur, -dür,-tır, -tir, -tur, -tür affiksi egaga qo'shilib kelganida, undan anglashilgan ma'noni kuchaytirib, ta'kidlab ko'rsatish uchungina xizmat qiladi. Demak, stilistik maqsadlarda qo'llanuvchi bu so'zshaklini eganing alohida turi, deb farqlashga asos yo'q. Masalan, quyidagi gapning egasi tarkibida qatnashgan -tir ko'rsatkichi orqali egadan tushunilgan narsa, hodisaning me'yordan ortiqligi, davomli ekanligi anglashilgan:

Daha geçen sonbaharda içime bir ateştir düştü(R.N.G.).

O`tgan kuzdayoq ichimda shunday otash yona boshladiki.

\section{Xulosa}

O'zbek tilshunosligidagi va turk tilshunosligidagi ega talqinlarining tahlili quyidagi xulosalarga kelindi:

O'zbek tilining an'anaviy grammatikasiga ko'ra gapning bosh bo'lagi sifatida ega qabul qilingan bo'lsa-da, keyingi paytlarda gapning bosh bo'lagi kesim degan nuqtai nao'ardan ish olib borilmoqda. Turk tilshunosligida esa, aksincha, kesim gapning tamal toshi, asosiy bo'lagi hisoblanadi. Turk tilshunosligida kesimni faqat ifoda materialiga ko'ra tasniflash masalasiga birmuncha e'tibor qilinganini ko'rish mumkin. Kesimning tuzilishiga ko'ra ikki so'zli kesim, takroriy kesim kabi turlari ajratilgan bo'lsa-da, bu tasniflar talabga to'la javob bermaydi. Demak, turk tilidagi kesim va uning spetsifik xususiyatlariga oid fikr-mulohazalarni jamlash hamda tahlil etish asnosida ma'lum bo'ladiki, bu gap bo'lagining izchil tadqiqi amalga oshirilmagan. O'zbek tilida eganing leksik-semantik xususiyatlariga ko'ra, tuzilishiga ko'ra tasniflari mavjud. Turk tilshunosligida ham eganing o'n, o'n bir turini qamrab oluvchi tasniflar amalga oshirilgan. Ammo turk tilidagi bu tasniflarda izchillikka rioya qilinmaganligi, sintaktik, stilistik va morfologik kategoriyalar qorishtirib yuborilganligi yaqqol ko'zga tashlanadi. Natijada, aslida eganing turi sifatida ajratilish uchun yetarli asos bo'lmagan hollarda ham bunday tasnif amalga oshirilganligini ko'ramiz.

Solishtirilayotgan tillardagi bosh bo'laklar tuzilishiga ko'ra uch xil bo'ladi: sodda, tarkibli, murakkab. Sodda ega va sodda ot-kesim ot, olmosh, otlashgan sifat, otlashgan sifatdosh, jamlovchi son yoki otlashgan son, ravish, harakat nomi kabi so'z turkumlari bilan ifodalanadi. Sodda bosh bo'laklar o'rtasidagi asosiy farqlar morfologik planda namoyon bo 'ladi. Masalan, turk tilida predmetning miqdorini, tartibini bildiruvchi so'zlar sifatning ma'no turlaridan biri tarzida, ya'ni son sifatlari deb o'rganiladi. Xususan, o'zbek tilida jamlovchi sonning yasovchilari bo'lmish, -ov, -ovlon, -ala affikslarining muqobillari turk tilida yo'q. Bu vaziyatda sanoq sonlarga murojaat qilinadi. Yoki: o'zbek tilidagi maqsad maylining -moqchi ko'rsatkichini olgan fe'llar sodda fe'l-kesim bo'lib keladi. Turk tilida ish-harakatning bajarish maqsadini ifodalash uchun kelasi zamon fe'lining ko'rsatkichi (-(y)ecek $\left.{ }^{2}\right)$ ga yoki «-mek affiksli harakat nomi + üzere /ustida (uchun) ko'makchisi» shaklli fe'lga murojaat qilinadi, bunda ikkinchi qayd etilgan ko'rinishdagi kesim tarkibli kesim hisoblanadi.

O'zbek tilidan farqli holda, turk tilshunosligida modal so'zlar alohida so'z turkumi sifatida farqlanmagan. Umuman, modal so'zlar sirasiga kiruvchi so'zlarning biron so' $z$ turkumi guruhiga kiritilgan hollari turk tilshunosligiga oid ilmiy manbalarda uchramadi. Lekin modal

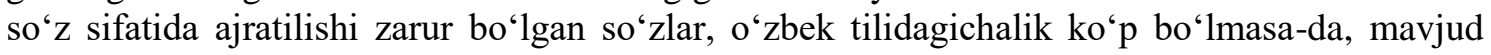
hamda bu so'zlar kesim vazifasini bajarib kelishi mumkin. Shuningdek, har ikki tildagi ega vazifasini bajaruvchi harakat nomi, sifat-doshning morfologik ko'rsatkichlari o'zaro anchagina tafovutlanadi. Xususan, o'zbek tilidan farqli ravishda turk tilidagi ega vazifasini bajargan so'zlar - dir $^{8}$ affiksini olgan bo'lishi mumkin. -dir affiksi egaga qo'shilib kelganida, undan anglashilgan ma'noni kuchaytirib, ta'kidlab ko'rsatish uchungina xizmat qiladi, ya'ni stilistik maqsadlar uchun qo'llaniladi. 
O'zbek va turk tillarida yetakchi fe'l hamda ko'makchi fe'ldan tashkil topgan fe'lning analitik shakli gapda tarkibli bosh bo'lak vazifasini bajaradi. O'zbek tilidagi fe'llar leksiksemantik xususiyatlariga ko'ra tasniflangani holda, turk tilshunosligida fe'llarni ma'nosiga ko'ra guruhlashtirishda izchillikning yo'qligi ko'zga tashlanadi. Shu bois, turk tilidagi fe'llarni o'zbek tilidagi fe'llar singari leksik-semantik xususiyatlariga ko'ra tasniflash maqsadga muvofiq.

O'zbek va turk tillaridagi «etakchi komponent + yordamchi fe'l» tipidagi fe'llarni o'rganish natijasida ma'lum bo'ldiki, o'zbek tilida yordamchi fe'lning barcha ma'no turlari ko'makchi, yordamchi, to'liqsiz fe'llarning tarkibli ega tarkibida faol ishtirok etishi kuzatiladi. Turk tilida esa asosan yordamchi fe'llar qatnashuvidagi tarkibli egalar qo'llanilishi ko'rinadi. Ega tarkibida ishtirok etgan to 'liqsiz fe'llar uchun yordamchi fe'llar ishlatiladi.

O'zbek va turk tillaridagi ikki komponentli tarkibli kesimlarning asosiy ko'rinishi «ot, ot xarakteridagi so' $z+$ yordamchi fe'l», «ot, ot xarakteridagi so' $z+$ to'liqsiz fe'l», «etakchi fe'l+to'liqsiz fe'l», «etakchi fe'l+ko'makchi fe'l» qolipiga egadir. Turk tilida bu qoliplarning asosan birinchi uch ko'rinishi keng tarqalgan bo'lib, «etakchi fe'l+ko'makchi fe'l» ko'rinishi nisbatan ayrim hollarni hisobga olmaganda, nofaoldir. Bu holat o'zbek tilidagi ko'makchi fe'llarning miqdori turk tilidagi ko'makchi fe'llarga nisbatan ko'pligi hamda ularning faol qo'llanilishi bilan belgilanadi.

Turg'un, barqaror ismlar, geografik, onomastik atamalar, tashkilot, muaassasa, kitob, jurnal nomlari, murakkab so'zlar, frazeologik birikmalar, aniqlovchili birikmalar, polupredikatlar, teng bog'lanishli qo'shilmalar, muayyan qolipli birikmalar solishtirilayotgan tillarda murakkab bosh bo'laklar vazifasini bajarib keladi.

\section{Bibliografiya}

ABDUllaYEV, H. (1969). Hozirgi O'Zbek Adabiy Tilida Gap Bo'Laklarining Ajralmas Sintaktik Konstruksiya Bilan Ifodalanishi. Filol.Fan.Nomz....Dis. Samarqand: Samarqand Davlat Universiteti.

ABDURAHMONOVA, M. (2004). O'Zbek Va Turk Tillarida Ot Kategoriyalarining Talqini. Filol.Fan.Nomz....Dis.Avtoref. Toshkent: O‘Zbekiston Milliy Universiteti.

ABDURASULOV, Yo. (2008). Turkiy Tillarning Qiyosiy-Tarixiy Grammatikasi. Toshkent, Fan.

BANGUOĞLU, T. (2004). Türkçenin Grameri. Ankara:Türk Tarih Kurumu Basım Evi.

BASKAKOV, N.A. (1960). Tyurkskiye Yazıki. Moskva: Nauka.

BILGIN, M. (2006). Anlamdan Anlatıma Türkçemiz. Ankara: Anı Yayıncılık.

BOLULU, O. (1992). Türkçede Eylemin ve Gerçekleştiricinin Özellikleri. Eylemler ve Özneler Üzerinde Bir Anlam İncelenmesi. Türk Dili, 31, 32, s. 24-29, 46-52.

DEMIR, N.; Y1lmaz, E. (2003). Türk Dili. Ankara: Grafiker Yayınc1lık.

DEMIRCAN, Ö. (2003). Türk Dilinde Çatı. İstanbul: Papatya.

DIZDAROĞLU, H. (1976). Tümcebilgisi. Ankara: TDK.

ERDAL, M. (1991). Old Turkic Word Formation. Otto Harrassowitz, Wiesbaden.

ERGIN, M. (1993). Türk Dili Bilgisi. İstanbul: Bayrak. Basımevi.

HATiBOĞLU, V. (1982). Türkçenin Söz Dizimi. Ankara: Ankara Üniversitesi

KARAHAN, L. (2006). Türkçede Söz Dizimi. Ankara: Akcağ. 
KORKMAZ, Z. (2003). Türkiye Türkçesinin Grameri. Ankara: TDK.

O'zbek tili grammatikasi. (1975). Ikki jildlik. J. I. Morfologiya. Toshkent: Fan.

OMONTURDIYEV, J. (1988). Hozirgi O'Zbek Adabiy Tilida Gap Bo'Laklari Tipologiyasi. Toshkent: O`qituvchi.

SAFAYEV, A. (1958). Glavnie Chlenı Predlojeniya V Sovremennom Uzbekskom Yazıke. Tashkent: Izd.SAGU.

SAYFULLAYEV, A.R. (1984). Semantiko-Grammaticheskiye Osobennosti Chlenov Predlojeniya V Sovremennom Uzbekskom Literaturnom Yazıke. Toshkent: Fan.

SEREBRENNIKOV, B.A., GADJIYEVA, N.Z. (1986). Sravnitelno-Istoricheskaya Grammatika Tyurkskix Yazıkov. Moskva: Nauka.

SEREBRENNIKOV, B.A., GADJIYEVA, R.Z. (1979). Sravnitelno-Istoricheskaya Grammatika Tyurkskix Yazıkov. Baku: Maarif.

SHABANOV, J. (2004). Turk Va O'Zbek Tillarida Harakat Fe'llari Fe'llari Semantikasi. Filol.Fan.Nomz....Diss. Toshkent: O’Zr FA Til Va Adabiyot Instituti. Nauka.

SHCHERBAK, A.M. (1970). Sravnitelnaya Fonetika Tyurkskix Yazıkov. Leningrad:

SHCHERBAK, A.M. (1977). Ocherki Po Sravnitelnoy Morfologii Tyurkskix Yazikov (Imya). Leningrad: Nauka.

SHCHERBAK, A.M. (1981). Ocherki Po Sravnitelnoy Morfologii Tyurkskix Yazıkov (Glagol). Leningrad: Nauka.

SHOAZIZOV, Sh. (1973). Hozirgi O'Zbek Adabiy Tilida Ega Va Uning Ifodalanishi. Filol.Fan.Nomz....Diss. Toshkent: O`Zr FA Til Va Adabiyot Instituti.

SIDDIQOV, Z. (2000). O'Zbek Va Turk Tillarida Ko'Ruv Leksemalari Semantikasi. Filol.Fan.Nomz...Dis.Avtoref. Toshkent: O`Zr FA Til Va Adabiyot Instituti.

Sravnitelno Istoricheskaya Grammatika Tyurskix Yazlkov. (Morfologiya). (1988). Moskva: Nauka.

Sravnitelno-Istoricheskaya Grammatika Tyurskix Yazıkov (Fonetika). (1984). Moskva: Nauka.

TUNA, O. N. (2003). Türkçede Geçişli Ettirgen Fiiller. TDED.XXIY-XXY. s.381-428.

UNDERHILL, R. (1976). Turkish Grammer. The MIT press.

USMONOVA, Sh. (1998). O'Zbek Va Turk Tillarida Somatik Frazeologizmlar. Filol.Fan.Nomz....Dis.Avtoref. Toshkent: O'Zbekiston Milliy Universiteti.

XUDOYBERGANOVA, Z. (1999). O'Zbek Va Turk Tillarida Fe'lning O'Tgan Zamon Shakllari Tizimi. Filol.Fan.Nomz.... Dis. Toshkent: O`Zr FA Til Va Adabiyot Instituti.

XUDOYBERGANOVA, Z. (2007). O'Zbek Va Turk Tillarida Monotaksemalarning Ilmiy Talqini. Filol.Fan.Nomz.... Dis. Toshkent: O`Zr FA Til Va Adabiyot Instituti.

YAMAN, E. (2000). Türkiye Türkçesi ve Özbek Türkçesinin Söz dizimi Bakımından Karşılaştırılması. Ankara: TDK. 